\title{
Fast Track Colorectal Surgery
}

\author{
Timothy C. Counihan, M.D. ${ }^{1,2}$ and Joanne Favuzza, D.0. ${ }^{1}$
}

\section{ABSTRACT}

A "fast track" colon surgery program is the global package of perioperative care encompassing preoperative, operative, and postoperative techniques, which in aggregate result in fewer complications, a reduction in cost, less postoperative pain, a reduction in the hospital length of stay, and quicker return to work and normal activities. Results of fast track programs have shown significant advantages; however, strong evidence is forthcoming. Implementation of a fast track program requires a significant commitment and a multidisciplinary approach. Fast track principles may also be applied to anorectal surgery with good results.

KEYWORDS: Fast track, recovery, pain reduction

Objectives: On completion of this article, the reader should be familiar with optimizing perioperative management and the fast track in colon and rectal surgery.

\section{COLON SURGERY}

The goal of having a swift, complication-free recovery for patients after colon and rectal surgery has existed as long as our profession. However, traditions and dogma abound in this field as in others. Traditional beliefs about the perioperative care of colorectal surgery patients have been challenged by recent study. In this article, we will evaluate the evidence currently available for both colon surgery and anorectal surgery and outline the perioperative care of patients that results in the least amount of complications and the quickest recovery.

The term "fast track" in colon surgery was first used by Professor Henrik Kehlet. ${ }^{1}$ Originally concerned primarily with patients' pain and length of stay, it has evolved to mean different things to different parties. Surgeons, patients, and third-party payers all have an idea of an "ideal" length of stay that may not be the same. ${ }^{2}$ For our purposes we will define fast track colon surgery as the global package of perioperative care encompassing preoperative, operative, and postoperative techniques, which in aggregate result in fewer complications, a reduction in cost, less postoperative pain, a reduction in the hospital length of stay, and quicker return to work and normal activities. In this first section, we will review the evidence in each area of concern. Then, the development of a program combining these elements with successful implementation will be addressed.

\section{Perioperative Anesthetic Care and Multimodal Analgesia}

As with fast track surgery principles, the concept of evidenced-based anesthetic care and multimodal analgesia is really a philosophic approach to care. Each of the components of these approaches has been studied with good effect, but the sum of the parts seems to provide even better results. A truly integrated perioperative medical management model incorporates preoperative, intraoperative, and postoperative evidence-based techniques that optimize patient outcomes. ${ }^{3}$
${ }^{1}$ Department of Surgery, Berkshire Medical Center, Pittsfield, Massachusetts; ${ }^{2}$ University of Massachusetts Medical School, Worcester, Massachusetts.

Address for correspondence and reprint requests: Timothy $\mathrm{C}$. Counihan, M.D., Department of Surgery, Berkshire Medical Center, 725 North St., Pittsfield, MA 01201 (e-mail: tcounihan@bhs1.org).
Perioperative Management and Anesthesia; Guest Editor, W. Brian Sweeney, M.D.

Clin Colon Rectal Surg 2009;22:60-72. Copyright (C) 2009 by Thieme Medical Publishers, Inc., 333 Seventh Avenue, New York, NY 10001, USA. Tel: +1(212) 584-4662.

DOI 10.1055/s-0029-1202888. ISSN 1531-0043. 


\section{PREOPERATIVE ISSUES}

To accomplish fast track goals, several issues need to be addressed. Patient selection is a critical and sometimes overlooked step. The anesthesiologist needs to identify patients suitable for the fast track approach. Once chosen, patients require significant teaching and much of this educational program can be performed in conjunction with the preoperative anesthesia visit. The continuation of medicines the patient is already taking, especially $\beta$ blockers and other medicines that blunt catecholamine release must be reinforced. There may even be a role for starting patients on $\beta$ blockers to improve outcomes. ${ }^{4}$ Continuing usual medicines up to and after surgery has been shown to reduce complications in at least one study.

\section{PERIOPERATIVE ISSUES}

Fluid Management Liberal fluid use during and after colon surgery has been a cornerstone of perioperative care of colon surgery patients. Fluid loading to counteract the mechanical bowel prep and overnight fast is followed by bolusing during induction, especially when using an epidural catheter. Replacement for third space losses during surgery and additional fluids should urine output or blood pressure drop are also given. The effect of this approach is a 3 to $6 \mathrm{~kg}$ weight gain, ${ }^{6}$ with a resultant potential delay in gastrointestinal function and at least theoretical increase in complications. With increasing concern that fluid overload is detrimental ${ }^{7}$ extensive study in patients with hip fractures has concluded fluid restriction in those patients reduces complications and mortality. ${ }^{8}$ Colectomy patients have not been studied as extensively and available data have been contradictory. One randomized trial involving $172 \mathrm{pa}-$ tients undergoing colectomy showed a reduction in complications with a more restrictive fluid regimen. ${ }^{9} \mathrm{~A}$ second study looking at gastrointestinal surgery patients, primarily colectomies, also showed a benefit to fluid restriction. ${ }^{10}$ However, other studies have failed to show a benefit. ${ }^{11}$ Although the ideal fluid regimen for colectomy patients has yet to be defined, fast track proponents argue for a more cautious approach to fluid resuscitation termed "goal-directed fluid administration."12

Normothermia It has been well established that patients lose body heat during colon surgery; this loss of heat results in several unwanted effects. ${ }^{13}$ The physiologic effects of hypothermia on the stress response has also been well described. ${ }^{14}$ Although the prime interest in controlling temperature has been around reduction in wound infection, data on patients in one well-designed randomized trial on colectomy patients showed beneficial effects on length of stay even in patients that were not infected. ${ }^{15}$ Because of these findings, aggressive control of patient temperature during colon surgery has become the standard of care. Patient normothermia has been made a core quality measure in the Surgical Care Improvement Program, the collaborative between the Center of Medicare and Medicaid Services and nine other healthcare organizations in the United States. ${ }^{16}$

Intravenous Lidocaine The beneficial effects of epidural analgesia using local anesthetic may be at least partially due to the systemic effects of these agents. Rimback et $\mathrm{al}^{17}$ initially tested this hypothesis by studying the effects of lidocaine on gastrointestinal motility, which showed a positive effect on motility at typical serum levels in human subjects. Groudine et $\mathrm{al}^{18}$ followed this work with a randomized trial showing a dramatic positive effect of intravenous (IV) lidocaine on postoperative pain, ileus, and length of stay in major urologic procedures. The only study done on colectomy patients was a randomized trial in patients undergoing laparoscopic colectomy. The study showed a salutary effect with regards to pain control, pulmonary function, and ileus. ${ }^{19}$ This study did not have patients who received epidural catheters, so it remains to be seen if there is a benefit greater for intravenous or epidural local anesthetics. Given the dramatic difference in cost of IV lidocaine (under \$3) versus the cost and time commitment of an epidural catheter, one could argue this topic demands further aggressive study. The use of IV lidocaine should be strongly considered in patients without epidural catheters.

Anesthetic Technique, Neuraxial Blockade, and the Use of Epidural Anesthesia/Analgesia Anesthesia that minimizes surgical stress will contribute to the fast track goals. The reduction of the body's response to surgical stress has been one of the main principles of the fast track approach to colon surgery. The response to surgical stress occurs through several pathways, but certainly the most important being the afferent nervous stimulation of the hypothalamus and anterior pituitary gland leading to the production of catecholamines and glucocorticoids. ${ }^{20}$ Neuraxial blockade has been shown in several studies to reduce the surgical stress response. In several fields an overall reduction in mortality and complications has been observed. ${ }^{21}$ Although there has been no large randomized trial to date showing such great benefits specifically in colon surgery patients, many of the benefits seen in the fast track literature may be directly due to the use of epidural catheters as part of the anesthetic plan. The use of epidural catheters has also become increasingly popular in the management of postoperative pain after colon surgery. Epidural analgesia has been shown to be superior to intravenous narcotics in controlling postoperative pain in open colon surgery. $^{22}$ This benefit has been shown in patients undergoing laparoscopic colon surgery as well. ${ }^{23}$ In 
addition to providing superior pain control, which in itself is one of the major goals in the fast track plan, epidural analgesia has been shown to reduce ileus. Jorgensen et $\mathrm{al}^{24}$ conducted a Cochrane Review concluding that epidural catheters using local anesthetic reduce postoperative ileus. Again, this benefit has been confirmed even in laparoscopic surgery patients. ${ }^{25} \mathrm{~A}$ third potential benefit of epidural catheter use is the reduction of other complications related to major surgery. The blockade of the neuroaxial system after surgery had been shown to improve outcomes in many other surgical patients. ${ }^{26}$ In fact, one meta-analysis showed an overall 30\% reduction in mortality and dramatic reductions in other complications. ${ }^{21}$ Although there is not a great amount of evidence specifically in colon surgery patients, leaders in the fast track field feel strongly that epidural analgesia contribute significantly to fast track benefits. ${ }^{3,27}$ Because of the many presumed and real benefits of epidural local anesthetics, this technique should be considered one of the cornerstones of any fast track program.

\section{POSTOPERATIVE ISSUES}

Postoperative Pain Postoperative pain is a major factor in the recovery of patients after colon surgery and is one of the principle contributing factors as to why patients stay in the hospital after surgery. Traditionally, patients were given intermittent doses of intramuscular or subcutaneous narcotics, which often provided inadequate pain control limiting patient ambulation. As described above, administration of thoracic epidural local anesthetic is a key facet in fast track colon surgery care, but epidural analgesia is just one part of a multimodal approach to pain management in these patients.

Patient-Controlled Analgesia Despite the evidence of the benefit of epidural catheter use, there are factors that make their use unpopular at some centers and alternatives for postoperative pain control are used. IV narcotics delivered by patient-controlled analgesia (PCA) devices have gained widespread acceptance. Proponents of PCA use in the context of fast track colon surgery have mostly focused on the reduced pain and reduced ileus effects of using a minimally invasive approach. ${ }^{28,29}$ These authors have not found additional benefit of epidurals beyond the laparoscopic approach and fast track plan. ${ }^{23,25} \mathrm{PCA}$ use in these patients has resulted in good pain control along with the usual fast track effects. Because the incisions are small, the total volume of narcotics required is modest. PCAs are also helpful in patients who refuse or who have contraindications for epidural catheters.

Nonsteroidal Antiinflammatory Drugs The use of nonsteroidal antiinflammatory drugs (NSAIDs) for postoperative pain is well established. Although concerns about bleeding and other complications ${ }^{30}$ have limited their use by some, a review of many studies ${ }^{31}$ shows them to be safe for short-term use. Specifically, ketorolac has been shown to reduce narcotic requirement and can be given prior to the return of bowel function. Oral NSAIDs may be used once bowel function returns as well. Proponents of NSAID use point out the utility of providing preemptive analgesia either preoperatively or intraoperatively for best results. ${ }^{31}$ In colectomy patients, ketorolac has been shown to reduce narcotic usage and lead to faster to return of bowel function. ${ }^{32}$ Given the benefits of reduced narcotic usage, NSAID use should be considered as part of a fast track regimen.

Wound Pumps Another way to use local anesthetics to control the stress response and reduce postoperative pain without narcotics is to infuse local anesthetic directly into the wound. Originally described in $1935,{ }^{33}$ this technique has been experimented with throughout the $20^{\text {th }}$ century and has been found to be safe and effective in several settings. ${ }^{34,35}$ Wound pump systems are now commercially available for use. Baig et $\mathrm{al}^{36}$ reported dramatic reduction in narcotic use in patients undergoing colectomy. A Cochrane Review has confirmed the efficacy of these devices in a variety of settings including abdominal surgery. ${ }^{37}$ Data are not currently available using local perfusion of anesthetics within the setting of a fast track program. However, reduction of narcotic usage is one of the principles of fast track programs, so it would be reasonable to incorporate wound pumps into fast track protocols especially in patients who do not have epidural catheters.

Postoperative Nausea and Vomiting Postoperative nausea and vomiting (PONV) are common unwanted side effects of general anesthesia. Several medications have been associated with the reduction in PONV. ${ }^{38}$ Supplemental oxygen has been shown to have a clear benefit in reducing PONV as well. Goll et al ${ }^{39}$ studied 240 patients in a randomized trial and concluded that 2 hours of $80 \%$ oxygen was more effective and less expensive then ondansetron in reducing PONV. The best evidence currently available is from a trial involving 5199 patients enrolled in a variety of protocols using dexamethasone, ondansetron, droperidol, propofol (instead of volatile agents), ultra-short-acting narcotics, supplemental oxygen, and avoiding nitrous oxide. This large study showed no particular advantage of one drug over another, and that more than one intervention (e.g., droperidol plus avoiding nitrous oxide) was more effective than a single intervention. When patients were stratified for risk, the effects were even more pronounced. ${ }^{40}$ There is currently no consensus regarding the exact regimen to follow ${ }^{3,41}$; however fast track advocates all agree that the anesthesia team needs to 
make a strong commitment to reducing PONV. ${ }^{1}$ Given these data, a multimodal approach to controlling PONV should be part of a fast track program. The approach should include intraoperative techniques such as avoiding nitrous oxide and the use of volatile agents, as well as including supplemental oxygen and an agent such as droperidol or dexamethasone.

\section{The Role of the Surgeon in Fast Track Colon Surgery}

\section{PREOPERATIVE ASSESSMENT, TEACHING, AND PREPARATION}

Proper assessment of patients prior to undergoing surgical procedures is a critical step to ensuring good outcomes. For the fast track plan to work, appropriate patients need to be identified and inappropriate patients excluded from the plan. Typical fast track programs exclude ASA IV patients, urgent procedures, and cases requiring extensive adhesiolyis. ${ }^{42}$ Other factors such as extreme age, need for inpatient rehabilitation, and low socioeconomic status ${ }^{43}$ can all effect timely discharge from the hospital and should be addressed before surgery. Although initially the fast track concept included the most routine operations, typically only right and sigmoid colectomy, it has been shown that more complex patients and procedures also are amenable to this approach. Delaney et $\mathrm{al}^{44}$ reported a reduction in length of stay among patients who underwent complex pelvic and abdominal procedures such as total proctocolectomy and abdominoperineal resection.

Proper education of patients prior to surgery is an important step to having a successful fast track program. $^{27,45}$ Some programs even require a specially trained nurse to deliver the preoperative education. ${ }^{42,44}$ The exact content of patient education is rarely spelled out in the literature and varies from program to program, but certain components are probably critical. Patients need to be counseled as to the fast track concept, expectations for pain management, expected milestones of each postoperative day, and expected discharge day.

\section{PERIOPERATIVE NUTRITION}

Preparing the patient nutritionally for the surgical stress is a new concept. Traditionally, patients fasted prior to colon surgery and underwent an extensive mechanical cleansing of the colon lasting one or more days. Recently, mechanical bowel preparation has been shown in multiple randomized trials and in a Cochrane Review to be unnecessary and perhaps harmful. ${ }^{46}$ Emerging evidence about the role of glucose metabolism and the body's response to stress may force us to reexamine our approach to preoperative nutrition. It is well established that insulin resistance is a result of surgical stress. ${ }^{47}$ It is also accepted that these changes in insulin metabolism are related to worse clinical outcomes. ${ }^{48}$ What has not been well established to date is whether modifying insulin resistance can have an impact on outcomes. Animal studies ${ }^{49}$ and small clinical trials have shown administration of glucose solutions prior to surgery is safe $^{50}$ and may have a beneficial effect on outcomes. ${ }^{51,52}$ More study is needed in this promising area.

\section{SURGICAL TECHNIQUE}

Since the advent of minimally invasive approaches to colon surgery in the early 1990s, advocates have touted that the laparoscopic approach to colon surgery is the most important factor in effecting the fast track approach to colon surgery. This is not to say that the fast track approach cannot be applied to open surgery, but some feel that the basic principles of the fast track approach are best accomplished using the laparoscopic approach. Proponents of laparoscopic colon surgery point to the reduction in surgical stress associated with this approach, ${ }^{53}$ and this is presumed to contribute to reduced ileus and other benefits. Preservation of pulmonary function is also a potential advantage to the laparoscopic approach. Schwenk et $\mathrm{al}^{54}$ reported an improvement in pulmonary function and recovery after laparoscopic versus open colectomy; however, this was a small series and the effects were modest. Large incisions and lack of fast track treatment of patients in the control group may have exaggerated the benefits. One randomized trial looking specifically at laparoscopic versus open colectomy using fast track principles in both groups failed to find any difference in all of the usual parameters, suggesting that fast track care is equivalent to the potential benefits of laparoscopic colon surgery. ${ }^{55}$ Laparoscopic surgery in combination with a fast track regimen in the elderly has been studied specifically: Bardram et $\mathrm{al}^{56}$ reported a case series of 39 patients with a median age of 80 years showing elderly patients received similar benefits as others with this approach.

A Cochrane Review looking at the short-term benefits of laparoscopic colorectal resection concluded that there were indeed short-term advantages. Less pain, reduced ileus, preservation of normal pulmonary function, shorter length of stay, less wound complications, and better quality of life in the first month have all been borne out in multiple randomized trials. ${ }^{57}$ However, the authors of this review made important observations regarding these potential advantages. A relative poor quality of the study designs was noted, often favoring the laparoscopic approach. Furthermore, the majority of these trials treated open colectomy patients in the standard fashion, without the benefit of the fast track approach. The authors specifically concluded that there is not sufficient evidence to conclude laparoscopic colectomy is superior to open colectomy when optimal perioperative management is applied and they called for more study. 
In conclusion, the laparoscopic approach to colon surgery is seen as critical to some and not as important to other leaders in this field. Given the current consumer climate in the United States, patients who are good candidates should be offered the laparoscopic approach as part of an overall fast track strategy. The LAFA trial (laparoscopic and/or fast track multimodal management versus standard care) is a large multicentered trial being conducted in the Netherlands. Opened in 2006, it is designed to specifically answer the question of whether fast track concepts enhance recovery even after a laparoscopic approach. ${ }^{58}$

\section{EARLY FEEDING}

Appropriate management of the gut after colon surgery is critical to a successful fast track plan. Originally described by Levin in $1921,{ }^{59}$ the nasogastric (NG) tube came into widespread use throughout the $20^{\text {th }}$ century. The theoretical benefits of the NG tube were to reduce anastomotic leak, abdominal wound dehiscence, aspiration, and even length of stay. ${ }^{60,61}$ Indeed, the next generations of surgeons were reluctant to question the wisdom of greats like Wangansteen, Mayo, and Ochner. ${ }^{62}$ Patient discomfort and concern for increased risk of pulmonary and other complications led many investigators to question the utility of routine NG tube use after elective colon surgery. ${ }^{63-66}$ Ultimately, no fewer then 26 randomized trials were performed in the latter half of the $20^{\text {th }}$ century ${ }^{67}$; two meta-analysis studies confirmed that there is no role for their use in routine colon surgery. ${ }^{67,68} \mathrm{NG}$ tubes delay discharge and increase pulmonary complications ${ }^{69}$; thus, they need to be avoided in fast track colon surgery patients.

Beyond avoiding NG tube use, is it safe to feed patients and will this further enhance fast track recovery? Fears related to early feeding include the risk of abdominal distention threatening the wound closure, aspiration, and anastomotic leak. However, early feeding has several theoretical advantages. Recent studies of nitrogen and glucose metabolism have suggested a benefit to supplying nutrients enterally around the time of surgical stress. ${ }^{48,70}$ In a meta-analysis of 11 trials, enteral feeding via feeding tube or by mouth was shown to reduce anastomotic leak, wound and other infections, pneumonia, and mortality. There was an associated reduction of length of stay as well. ${ }^{71}$ Recently, a Cochrane Review confirmed the findings that there is no benefit to withholding enteral nutrition in colectomy patients. ${ }^{72}$ In conclusion, early feeding after colon surgery is an important part of any fast track program.

The use of mu opioid receptor antagonists has also been shown in several studies to reduce postoperative ileus. ${ }^{73-75}$ Although it is not clear whether this benefit exists above the fast track approach, it is likely that this class of agent will be a component of fast track protocols. ${ }^{3}$

\section{AMBULATION/REHABILITATION}

Early ambulation of patients after colon surgery is a universally accepted part of a fast track plan. Fast track proponents initially incorporated early ambulation without real evidence that it impacts recovery in colon surgery patients. ${ }^{56}$ Jakobsen et $\mathrm{al}^{76}$ studied the effects of early mobilization in a fast track program after patients were sent home and showed significant improvement in such parameters as fatigue, sleep, return to leisure activity and activities of daily living. Because the use of early ambulation has been so widely accepted, it is unlikely that there will be any further specific study in this area.

\section{Results of Using Fast Track Programs}

Given all of the above factors that each may contribute to the goals of fast track surgery, one would expect strong evidence of the utility of these programs. Results of clinical studies on patients in fast track colon surgery protocols have been reported since Kehlet ${ }^{1}$ introduced the concept. Retrospective studies, ${ }^{44,77}$ prospective single arm studies, ${ }^{22,57}$ and case control trials ${ }^{76,78,79}$ have shown major advantages in outcomes compared with traditional colon surgery patients. Because there is significant variability in the components of each fast track program, results are difficult to compare. Differences around the laparoscopic approach, use of epidural catheters, and method of postoperative pain control are just examples of how different these programs can be. Unfortunately, there is a paucity of large randomized prospective trials. A systematic meta-analysis of fast track surgery identified only three randomized trials and three case control trials that passed the Cochrane Center's rigid criteria for evidence-based medicine analysis. ${ }^{58}$ Even the six trials evaluated had discrepancies in endpoints and data collected. The conclusion of the authors was that further study was needed before endorsing the fast track concept in colon surgery. Potential components of a fast track program are shown in Table 1.

\section{Implementation of Fast Track Protocols}

An organized approach to instituting a fast track protocol is considered paramount to achieving optimal results by fast track proponents. ${ }^{1}$ Because of this, the steps to setting up a successful fast track program need to be outlined. However, one interesting counter to this premise deserves mention. Walter et $\mathrm{al}^{80}$ reported in a survey from the United Kingdom that many surgeons who do not consider themselves "fast track" surgeons practice one or more of the critical components to the fast track approach. The survey suggests that an alternative, insidious acceptance of fast track principles is at least possible without the effort of deploying a specific program per se. It can be assumed, however, that a 


\section{Table 1 Components of a Fast Track Program}

\begin{tabular}{l}
\hline Preoperative assessment and planning \\
Patient selection \\
Patient education \\
Anesthesia assessment and teaching \\
Intraoperative considerations \\
Thoracic epidural catheter using local anesthetic \\
Limit intravenous fluids \\
Maintain normothermia \\
Use of minimally invasive approach or small incision \\
Postoperative care \\
Supplemental oxygen \\
No nasogastric tube/early feeding (consider promotility agent) \\
Multimodal pain management (epidural, ketorolac, etc.) \\
limiting narcotics \\
Early ambulation \\
Discharge planning/case management beginning \\
post-operative day (POD) \#1
\end{tabular}

review of the recommended method to deploy a fast track program may be helpful to some.

In our current complex health care environment, any system changes require a multidisciplinary approach. Developing a fast track colon surgery program is no exception. One of the most important considerations before embarking is the commitment of the surgeons performing colon surgery at the hospital. If the surgeons are unlikely to change the way they manage their patients, any program is doomed to failure. Once "buy in" has been established, a surgeon champion needs to head this task force. If possible, a midlevel provider (nurse practitioner or physician's assistant) working with colon surgery patients should also be involved in this process, as they can be a critical resource across the health care continuum as the program is implemented. The task force needs to have representation from all the appropriate areas in the hospital as well as quality management. The potential members of a task force are listed in Table 2.

\section{Table 2 Membership of a Fast Track Colon Surgery} Task Force

Surgeon
Anesthesiologist
Nursing leadership from:
Pretesting
Operating room
Postanesthesia care unit
Surgical floor
Nursing educator
Case management
Pharmacist
Quality management
Medical records/information technology
Physical therapy

Surgeon

Anesthesiologist

Nursing leadership from:

Pretesting

Operating room

Postanesthesia care unit

Surgical floor

Nursing educato

Case management

Pharmacist

Medical records/information technology
Once formed, the initial work will involve several assessments. The group needs to identify best practice as it relates to fast track colon surgery (usually the surgeon will do this). The group needs to assess what is current practice and outcomes at their institution. This is best done by nursing/quality management survey of the surgeons and nursing staff. Included are any patient education processes, ambulation protocols, usual anesthesia practices, standardized orders, and case management processes. Quality management will need to supply length of stay and complication data. It may be desirable to carry out a prospective detailed analysis of a cohort of colon surgery patients to further define the current practice and outcomes. This data will be helpful to use as comparison once a program is established.

With the current state of colon surgery at the hospital defined, variance between current and best practice can be identified. The group will then be able to generate a list of specific goals for their fast track program. This of course will be different for each institution. After goals are identified, the appropriate team members can begin to work on action plans for implementing change. As action plans are identified, they are brought to the group and further refined.

Completion of the action plans can be challenging, as many of the goals identified will cut across practitioners and locations in the hospital. Nursing education programs, development of appropriate forms and order sheets, patient education materials, changes in case management processes, and development of postoperative protocols for feeding and pain control are all examples. After all of the goals have been addressed and action plans performed, the first iteration of your fast track program will be established. This program needs to be presented to the appropriate surgeons and anesthesia staff and input solicited. The same process should be performed with nursing staff as well.

Once input has been collected and incorporated into the program, the fast track program will need to be applied to patients. A robust education process to roll out the fast track program is necessary to ensure everyone caring for the patients is on the same page, and understands the goals and specific processes of your program. Patients enrolled will undergo all of the processes outlined: $100 \%$ concurrent review by the task force of these initial patients and compliance with the processes is highly recommended. There will be inevitable glitches and errors as the program starts. Nursing and physician leadership will need to be diligent at this stage to drive the change. Once the processes are being followed reliably, analysis of the initial cohort of patients is performed. Data collection by quality management or another identified individual (midlevel provider) should be standardized and predetermined by the task force. Data should include administrative 


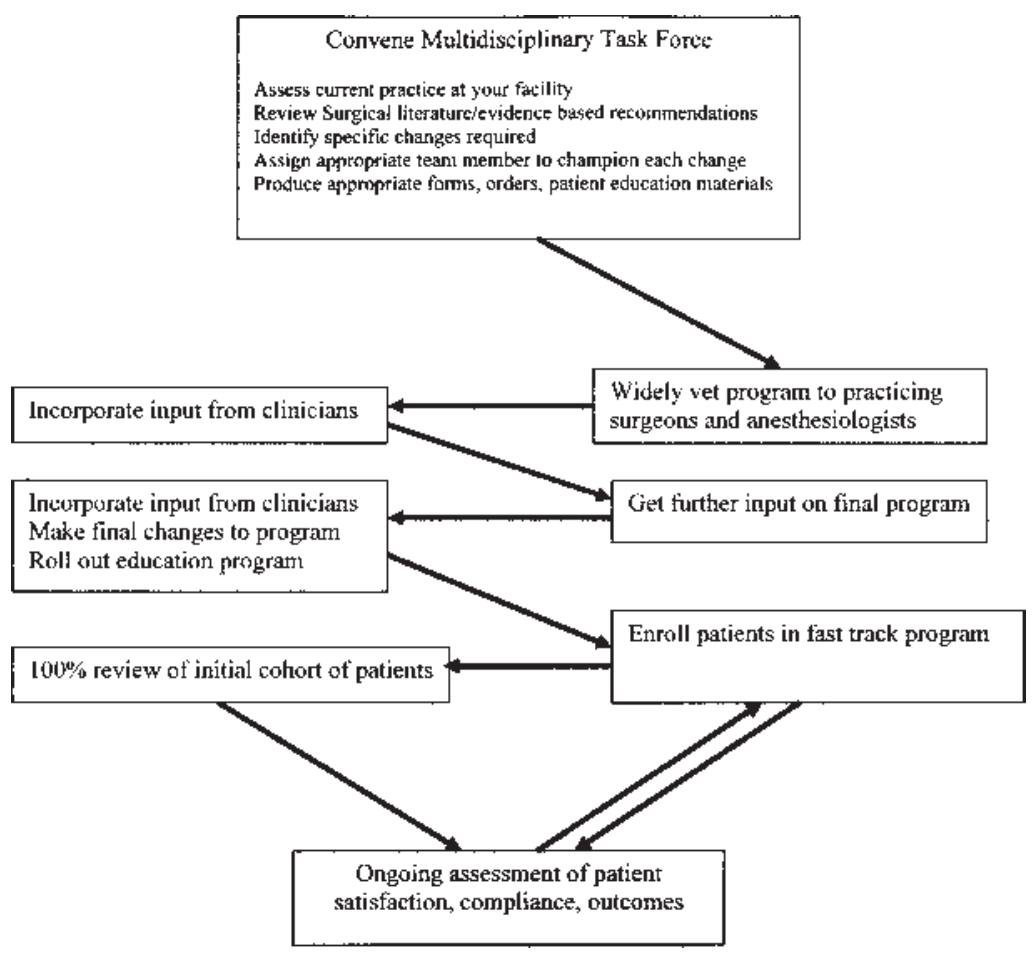

Figure 1 Developing a fast track program.

and financial data, process compliance data, outcomes, and patient satisfaction. Once presented to the task force, potential changes in the program should be entertained. Finally, clinical data confirming the safety and benefits of the new program is shared with the surgical staff.

At this point, ongoing management of the program will require tracking patient outcomes and process compliance. The task force can be dissolved and this function can be performed by quality management and the surgical champion of the program. A summary of this entire process is illustrated in Fig. 1.

\section{ANORECTAL SURGERY}

Anorectal disease occurs in $\sim 5 \%$ of the adult population in the United States, of which $10 \%$ will require operative intervention. ${ }^{81}$ Despite concerns by some, anorectal surgery can be performed in the ambulatory setting most of the time. ${ }^{82}$ Ambulatory anorectal surgery has been successful for a multitude of anorectal conditions such as abscesses, condylomata, fissures, fistulas, hemorrhoids, pilonidal cysts, and early rectal tumors. ${ }^{83}$ Given the frequency of these procedures, the establishment of a fast track program would be beneficial as well as cost effective. As with colon surgery, the goal of the fast track approach to anorectal surgery is quick recovery with minimal complications. Fast track anorectal surgery promotes optimal anesthetic technique, postoperative pain control, and early mobilization and discharge.

\section{Anesthetic Techniques}

The goal for optimal anesthesia in fast track anorectal surgery includes adequate sedation and analgesia, rapid recovery, and few postoperative side effects allowing for early discharge to home. ${ }^{84}$ Ambulatory surgery can be performed using general, regional, and local anesthesia or a combination of these.

In ambulatory anorectal anesthesia, general anesthesia has the advantage of providing amnesia, analgesia, and muscle relaxation. However, the duration of standard general anesthetics will often exceed the length of the operation. ${ }^{85}$ The postoperative nausea and vomiting (PONV) following general anesthesia may result in a delay in recovery and discharge. ${ }^{86}$ The use of the shortacting intravenous anesthetic propofol, has been useful in the fast track care of patients. Propofol has ideal pharmacokinetics, specifically zero-order kinetics, which allows the anesthesiologist to match the infusion rate with level of desired sedation. ${ }^{87}$ When volatile anesthetics are necessary, the use of short-acting agents such as desflurane and sevoflurane, reduces recovery time and cost in patients. ${ }^{88}$ Such rapid-acting agents have the benefit of faster recovery.

Regional anesthesia provides analgesia through the direct administration to the spinal cord or nerves thereby locally blocking afferent and efferent nerve impulses. ${ }^{89}$ The local anesthetic is delivered into the subarachnoid space (spinal) or the epidural space. ${ }^{21}$ Spinal anesthesia is commonly used for ambulatory surgery due to short duration of time needed to perform the block. Spinal anesthesia also has a reliable sensory 
and motor block as well as decreased pain during surgery. ${ }^{90}$ Regional anesthesia avoids some of the complications of general anesthesia such as sore throat and airway trauma. ${ }^{85}$ In addition, patients typically have decreased postoperative nausea and vomiting, improved pain relief, and decreased recovery time. ${ }^{90} \mathrm{~A}$ metaanalysis comparing the use of regional anesthesia compared with general anesthesia alone has demonstrated a reduction in postoperative mortality with a significant decrease in pulmonary, cardiac, and thromboembolic complications with regional anesthesia. ${ }^{21}$ Despite these advantages, regional anesthesia can result in urinary retention and delayed ambulation secondary to slow recovery from motor blockade. ${ }^{84}$

Local anesthesia with sedation has recently increased in popularity with the improvement in sedation techniques. ${ }^{91}$ Local anesthetic techniques utilized are posterior perineal blocks and local anesthesia of the anal canal and perineal skin. ${ }^{85}$ With the posterior perineal block, first described by Marti, ${ }^{92}$ the anal canal is infiltrated with bupivacaine or lidocaine following the posterior perineal nerves. An alternative technique employs the use of an anoscope to anesthetize above the dentate line providing relaxation of the anal canal, which is followed by an injection below the dentate line for the perineal skin. ${ }^{85}$ The success of this technique relies on the technical skill of the surgeon to infiltrate the necessary areas effectively. Local anesthesia with sedation reduces the complications associated with general or regional anesthesia, such as postoperative nausea and vomiting and urinary retention. This technique has been associated with a reduction in the severity of postoperative pain allowing for decreased opioid use. ${ }^{81}$

One particular combination that has been shown to be useful for ambulatory anorectal surgery is the use of intravenous sedation with propofol/ketamine with local anesthesia. Ketamine is unique in that it provides analgesia while also working as a cardiovascular and respiratory stimulant. ${ }^{93}$ These advantages are particularly helpful for patients in the prone position. This combination has been reported to shorten the time in the hospital and reduce costs. ${ }^{93}$

One randomized trial compared the three anesthetic techniques in patients undergoing anorectal surgery. Patients were either assigned to local bupivacaine and lidocaine with propofol infusion, spinal subarachnoid block with lidocaine and fentanyl, or general anesthesia consisting of propofol, sevoflurane, and nitrous oxide. In this study, anesthesia time, time to oral intake, and time to discharge were shortest for patients who received local anesthesia with propofol. Patients in this group also had no episodes of nausea and did not require supplemental oxygen in the postanesthesia care unit. Overall, the use of local anesthesia combined with sedation was found to be superior to spinal and general anesthesia in terms of recovery, side effects, and cost effectiveness. ${ }^{81}$

\section{OPERATIVE TECHNIQUES}

One of the most common anorectal disorders requiring surgery is hemorrhoidal disease. ${ }^{94}$ Most hemorrhoidal surgery is performed as ambulatory surgery. Traditionally, excision of hemorrhoidal tissue with either the Milligan-Morgan (open) or Ferguson (closed) techniques has been performed under general anesthesia and has been associated with significant postoperative pain. ${ }^{95}$ The ideal fast track technique for hemorrhoid surgery would include an effective technique with minimal complications and the least postoperative pain. In 1998, Antonio Longo developed the stapled hemorrhoidectomy, also known as the procedure for prolapsed hemorrhoids (PPH), this technique employs a circular stapler that circumferentially resects mucosa and submucosa above hemorrhoids. ${ }^{96} \mathrm{~A}$ stapled anastomosis is formed in the rectum at least $3 \mathrm{~cm}$ above the dentate line, which is a relatively insensate area. ${ }^{97} \mathrm{With}$ this technique, also termed stapled hemorrhoidopexy, the redundant prolapsing hemorrhoidal tissue is repositioned into the anal canal. ${ }^{97} \mathrm{~A}$ systematic review of 25 randomized trials comparing PPH with conventional hemorrhoidectomy concluded that $\mathrm{PPH}$ resulted in less postoperative pain and faster recovery with shorter time off work and better wound healing. ${ }^{98}$ A Cochrane Review concluded that stapled hemorrhoidopexy was as safe as conventional hemorrhoidectomy. ${ }^{99}$

The Harmonic Scalpel (Ethicon Endo-Surgery, Johnson \& Johnson, Inc., New Brunswick, NJ) has been utilized in the treatment of grades II, III, and IV hemorrhoids. ${ }^{100}$ This device cuts as well as coagulates hemorrhoidal tissue using ultrasonic vibrations. ${ }^{101} \mathrm{~A}$ prospective trial comparing the harmonic scalpel to electrocautery hemorrhoidectomy demonstrated diminished pain. ${ }^{102}$ The decreased postoperative pain with the harmonic scalpel is most likely due to the minimal lateral thermal injury. ${ }^{103}$ Harmonic Scalpel hemorrhoidectomies performed under local anesthesia with sedation has contributed to shorter operative time and hospital stay with reduced cost. ${ }^{104}$ Another excision device the LigaSure vessel sealing system (Valleylab Inc., Boulder, CO) uses both pressure and electrical energy to coagulate blood vessels also with minimal thermal energy spread. ${ }^{105}$ In a randomized trial comparing the Harmonic Scalpel with LigaSure for hemorrhoidectomy, both had comparable complication rates, patient satisfaction, and hospital stay. ${ }^{106}$

\section{Postoperative Care}

\section{PAIN CONTROL}

One of the most important aspects of fast track surgery is adequate pain control. To achieve effective pain control, Kehlet and Wilmore proposed "multimodal analgesia," which involves the combination of multiple agents. ${ }^{1}$ The 
use of opioid analgesics together with nonopioid options provides the most successful pain control with the greatest patient satisfaction.

Opioid analgesics are often used for moderate to severe postoperative pain; however, the side effects of nausea, vomiting, constipation, urinary retention, and respiratory depression often delays discharge. ${ }^{107}$ Therefore, limiting the use of opioids is a goal of the fast track program.

Nonopioid drugs, such as NSAIDS, improve pain control and facilitate early discharge. ${ }^{108}$ Ketorolac, a common NSAID, has been found to decrease the utilization of opioid medications. ${ }^{109}$ Ketorolac used either intravenously or locally after anorectal surgery improved postoperative pain control. ${ }^{110}$ Local injection of ketorolac compared with oral narcotics has similar pain control, but with better patient satisfaction and no postoperative urinary retention. ${ }^{111,112}$

\section{POSTOPERATIVE NAUSEA AND VOMITING}

Control of PONV is necessary for the early discharge of patients. Postoperative nausea is a common side effect of general anesthesia. Use of prophylactic antiemetic drugs during general anesthesia can decrease PONV, which will increase patient satisfaction. ${ }^{41}$ Droperidol has been shown to be cost effective and safe. ${ }^{107}$ If the patient is at high risk for PONV, then the addition of a serotonin antagonist has been shown to be effective. ${ }^{113}$ The use of dexamethasone given preoperatively has been found to be useful in decreasing nausea and vomiting. ${ }^{114,115}$ Betamethasone given to patients undergoing hemorrhoidectomies has been shown to decrease postoperative pain and nausea. ${ }^{116}$ Moreover, a multimodal approach to antiemetic treatment including adequate hydration has been shown to enhance patient approval. ${ }^{117,118}$

\section{URINARY RETENTION}

The most common complication following anorectal surgery is urinary retention, with a rate as high as $52 \% .{ }^{119}$ In patients undergoing anorectal surgery with spinal anesthesia, the risk factors contributing to urinary retention include excessive intravenous fluid administration and inadequate pain relief. ${ }^{120}$ To decrease the rate of urinary retention, intraoperative intravenous fluid restriction is the most important consideration. ${ }^{100}$ With fluid restriction the rate of urinary retention can be as low as 1.2 to $3.5 \% .^{121,122}$

\section{Recommendations for Fast Track Anorectal Surgery}

Fast track surgery is an efficient and cost-effective approach that can be applied to many common anorectal procedures. The first step involves choosing the most appropriate anesthetic agent, preferably local with seda- tion or regional anesthesia. The avoidance of general anesthesia will avoid many postoperative complications. In terms of operative techniques, fast track surgery may be applied using less invasive techniques such as PPH and the Harmonic Scalpel as mentioned with hemorrhoidal surgery. Postoperatively, limiting the use of opioids and optimizing the use of NSAIDS, like ketorolac both intravenously and locally should be actioned. Fluid restriction should be employed to decrease urinary retention postoperatively. Early ambulation also facilitates early discharge of patients. Fast track anorectal surgery can be safely performed with optimal anesthetic and pain control, minimal complications, early discharge, and reduced costs.

\section{REFERENCES}

1. Kehlet H, Wilmore DW. Multimodal strategies to improve surgical income. Am J Surg 2002;183:630-641

2. Schoetz DJ, Bockler M, Rosenblatt MS, et al. "Ideal" length of stay after colectomy: whose ideal? Dis Colon Rectum 1997;40(7):806-810

3. White PF, Kehlet H, Neal JM, Schricker T, Carr DB, Carli F. The role of the anesthesiologist in fast-track surgery: from multimodal analgesia to perioperative medical care. Anesth Analg 2007;104(6):1380-1396

4. Devereaux PJ, Beattie WS, Choi PT-L, et al. How strong is the evidence for the use of perioperative beta-blockers in patients undergoing noncardiac surgery? A systematic review and metaanalysis BMJ 2005;331:313-321

5. Kennedy JM, van Rij AM, Spears GF, Pettigrew RA, Tucker IG. Polypharmacy in a general surgical unit and consequences of drug withdrawal. Br J Clin Pharmacol 2000;49:353-362

6. Lobo DN, Bostock KA, Neal KR, et al. Effect of salt and water balance on recovery of gastrointestinal function after elective colonic resection: a randomized controlled trial. Lancet 2002;359:1812-1818

7. Lowell JA, Schifferdecker C, Driscoll DF, Benotti PN, Bistrian BR. Postoperative fluid overload: not a benign problem. Crit Care Med 1990;18(7):728-733

8. Price JD, Sear JW, Venn RM. Perioperative fluid volume optimization following proximal femoral fracture. Cochrane Database Syst Rev 2004;(1):CD003004

9. Brandstrup B, Tonnesen H, Beier-Holgersen R, et al. Effects of intravenous fluid restriction on postoperative complications: comparison of two perioperative fluid regimens: a randomized assessor-blinded multicenter trial. Ann Surg 2003;238:641-648

10. Nisanevich V, Felsenstein I, Almogy G, et al. Effect of intraoperative fluid management on outcome after intraabominal surgery. Anesthesiology 2005;103:25-32

11. Holte K, Foss NB, Andersen J. Liberal or restrictive fluid administration in fast track colonic surgery: a randomized, double-blind study. Br J Anaesth 2007;99(4):500-508

12. Holte K, Kehlet H. Fluid therapy and surgical outcomes in elective surgery: a need for reassessment in fast-track surgery. J Am Coll Surg 2006;202(6):971-989

13. Frank SM, Higgins MS, Breslow MJ, et al. The catecholamine, cortisol, and hemodynamic responses to mild 
perioperative hypothermia: a randomized clinical trial. Anesthesiology 1995;82:83-93

14. Sessler DI. Non-pharmacologic prevention of surgical wound infection. Anesthesiol Clin 2006;24(2):279-297

15. Kurz A, Sessler DI, Lenhardt R. Perioperative normothermia to reduce the incidence of surgical-wound infection and shorten hospitalization. N Engl J Med 1996;334(19):12091215

16. Joint Commission on Accreditation of Healthcare Organizations. Specifications Manual for National Hospital Quality Measures. Version 2.5 JCAHO. Oakbrook Terrace, IL: Joint Commission on Accreditation of Healthcare Organizations; 2008

17. Rimback G, Cassuto J, Tollesson P. Treatment of postoperative paralytic ileus by intravenous lidocaine infusion. Anesth Analg 1990;70:414-419

18. Groudine SB, Fisher HA, Kaufman RP Jr, et al. Intravenous lidocaine speeds the return of bowel function, decreases postoperative pain, and shortens hospital stay in patients undergoing radical retropubic prostatectomy. Anesth Analg 1998;86:235-239

19. Kaba A, Laurent S, Detroz B, et al. Intravenous lidocaine infusion facilitates acute rehabilitation after laparoscopic colectomy. Anesthesiology 2007;106:11-18

20. Wilmore DW. From Cuthbertson to fast track surgery: 70 years of progress in reducing stress in surgical patients. Ann Surg 2002;236:643-648

21. Rodgers A, Walker N, Schug S, et al. Reduction of postoperative mortality and morbidity with epidural or spinal anesthesia: results from and overview of randomized trial. BMJ 2000;321:1493-1504

22. Basse L, Hjort Jakobsen D, Billesbolle P, Werner M, Kehlet H. A clinical pathway to accelerate recovery after colonic resection. Ann Surg 2000;232:51-57

23. Senagore AJ, Delaney CP, Mekhail N, Dugan A, Fazio VW. Randomized clinical trial comparing epidural anaesthesia and patient-controlled analgesia after laparoscopc segmental colectomy. Br J Surg 2003;90:11951199

24. Jorgensen H, Wetterslev J, Moiniche S, Dahl JB. Epidural local anaesthetics versus opioid-based analgesic regimens for postoperative gastrointestinal paralysis, PONV and pain after abdominal surgery. Cochrane Database Syst Rev 2000;(4):CD001893

25. Neudecker J, Schwenk W, Junghans T, Pietsch S, Bohm B, Muller JM. Randomized controlled trial to examine the influence of thoracic epidural analgesia on postoperative ileus after laparoscopic sigmoid resection. Br J Surg 1999;86: 1292-1295

26. Holte K, Sharrock NE, Kehlet H. Pathophysiology and clinical implication of perioperative fluid excess. $\mathrm{Br} \mathrm{J}$ Anaesth 2002;89:622-632

27. Kehlet H, Wilmore DW. Evidence-based surgical care and the evolution of fast-track surgery. Ann Surg 2008;248(2): 189-198

28. Woodhouse A, Hobbes AFT, Mather LE, Gibson M. A comparison of morphine, pethidine and fentanyl in the postsurgical patient-controlled analgesia (PCA) environment. Pain 1996;64:115-121

29. Hong X, Mastraletti G, Zandi S, Stein B, Charlebois P, Carli F. Laparoscopy for colectomy accelerates restoration of bowel function when using patient controlled analgesia. Can J Anaesth 2006;53(6):544-550
30. Murray RP, Watson RC. Acute renal failure and gastrointestinal bleed associated with postoperative toradol and vancomycin. Orthopedics 1993;16(12):1361-1363

31. Souter AJ, Fredman B, White PF. Controversies in the perioperative use of nonsteroidal anti-inflammatory drugs. Anesth Analg 1994;79(6):1178-1190

32. Schlachta CM, Burpee SE, Fernandez C, Chan B, Mamazza J, Poulin EC. Optimizing recovery after laparoscopic colon surgery (ORAL-CS). Surg Endosc 2007;21: 2212-2219

33. Capelle W. Die bedentung des wundschmerzes und seiner ausschaltung fur dan ablauf der atmung bei laparotomierte. Dtsch Z Chir 1935;246:466-485

34. Patel JM, Lanzafame RJ, Williams JS, et al. The effect of incisional infiltration of bupivacaine hydrochloride upon pulmonary functions, atelectasis and narcotic need following elective cholecystectomy. Surg Gynecol Obstet 1983;157: 338-340

35. Cheong WK, Seow-Choen F, Eu KW, Tang CL, Heah SM. Randomized clinical trial of local bupivacaine perfusion versus parenteral morphine infusion for pain relief after laparotomy. Br J Surg 2001;88:357-359

36. Baig MK, Zmora O, Derdemezi J, Weiss EG, Nogueras JJ, Wexner SD. Use of the ON-Q pain management system is associated with decreased postoperative analgesic requirement: double blind randomized placebo pilot study. J Am Coll Surg 2006;202(2):297-305

37. Liu SS, Richman JM, Thirlby RC, Wu CL. Efficacy of continuous wound catheters delivering local anesthetic for postoperative analgesia: a quantitative and qualitative systematic review of randomized controlled trials. J Am Coll Surg 2006;203(6):914-932

38. Apfel CC, Kranke P, Eberhart LH. Comparison of surgical site and patient's history with a simplified risk score for the prediction of postoperative nausea and vomiting. Anaesthesia 2004;59(11):1078-1082

39. Goll V, Akca O, Greif R, et al. Ondansetron is no more effective than supplemental intraoperative oxygen for prevention of postoperative nausea and vomiting. Anesth Analg 2001;92:112-117

40. Carlisle JB, Stevenson CA. Drugs for preventing postoperative nausea and vomiting. Cochrane Database Syst Rev 2006;(3):CD004125

41. White PF, Song D. New criteria for fast-tracking after outpatient anesthesia: a comparison with the modified Aldrete's scoring system. Anesth Analg 1999;88:10691072

42. Behrns KE, Kircher AP, Galanko JA, Brownstein MR, Koruda MJ. Prospective randomized trial of early initiation and hospital discharge on a liquid diet following elective intestinal surgery. J Gastrointest Surg 2000;4:217-221

43. Junger M, Schoenberg MH. Postoperative care in fast track surgery. Transfusion Alternatives in Transfusion Medicine 2007;9:66-77

44. Delaney CP, Fazio VW, Senagore AJ, Robinson B, Halverson AL, Remzi FH. "Fast track" postoperative management protocol for patients with high co-morbidity undergoing complex abdominal and pelvic colorectal surgery. Br J Surg 2001;88:1533-1538

45. Egbert LD, Battit GE, Welch CE, Bartlett MK. Reduction of postoperative pain by encouragement and instruction of patients. A study of doctor-patient rapport. N Engl J Med 1964;270:825-827 
46. Guenaga K, Atallah AN, Castro AA, Matos DDM, WilleJorgensen P. Mechanical bowel preparation for elective colorectal surgery. Cochrane Database Syst Rev 2005;(1): CD001544

47. Black PR, Brooks DC, Bessey PQ, Wolfe RR, Wilmore DW. Mechanisms of insulin resistance following injury. Ann Surg 1982;196:420-435

48. Thorell A, Nygren J, Ljungqvist O. Insulin resistance: a marker of surgical stress. Curr Opin Clin Nutr Metab Care 1999;2:69-78

49. Aligobevic A, Ljungqvist O. Pretreatment with glucose infusion prevents fatal outcome after hemorrhage in food deprived rats. Circ Shock 1993;39:1-6

50. Nygren J, Thorell A, Jacobsson H, et al. Preoperative gastric emptying. Effects of anxiety and oral carbohydrate administration. Ann Surg 1995;222:728-734

51. Nygren J, Soop M, Thorell A, Efendic S, Nair KS, Ljungqvist O. Preoperative oral carbohydrate administration reduces postoperative insulin resistance. Clin Nutr 1998; 17:65-71

52. Gutniak M, Grill V, Efendic S. Effect of composition of mixed meals-low versus high-carbohydrate content-on insulin, glucagons, and somatostatin release in healthy human and in patients with NIDDM. Diabetes Care 1986;9(3):244-249

53. Kehlet $H$, Nielson HJ. Impact of laparoscopic surgery on stress responses, immunofunction, and risk of infectious complications. New Horiz 1998;6(2, suppl):S80-S88

54. Schwenk W, Bohm B, Witt C, et al. Pulmonary function following laparoscopic or conventional colorectal resection. Arch Surg 1999;134:6-12

55. Basse L, Jakobsen HD, Bardram L, et al. Functional recovery after open versus laparoscopic colonic resection. A randomized, blinded study. Ann Surg 2005;241:416-423

56. Bardram L, Funch-Jensen $P$, Kehlet H. Rapid rehabilitation in elderly patients after laparoscopic colonic resection. Br J Surg 2000;87:1540-1545

57. Schwenk W, Hasse O, Neudecker J, Muller JM. Short term benefits for laparoscopic colorectal resection. Cochrane Database Syst Rev 2005;(2):CD003145

58. Wind J, Polle SW, Fung Kon Jin PHP. Systematic review of enhanced recovery programmes in colonic surgery. Br J Surg 2006;93:800-809

59. Levin AI. A new gastrodenal catheter. JAMA 1921;76: 1007-1009

60. D'Costa H, Taylor EW. Patient management following uncomplicated elective gastrointestinal operations. Br J Clin Pract 1990;44:552-555

61. Jamieson WG, DeRose G, Harris KA. Routine nasogastric decompression after abdominal surgery? Can J Surg 1992; 35:577-578

62. Sagar PM, Kruegener G, MacFie J. Nasogastric intubation and elective abdominal surgery. Br J Surg 1992;79:1127-1131

63. Bauer JJ, Gelernt IM, Salky BA, Kreel I. Is routine postoperative nasogastric decompression really necessary? Ann Surg 1985;201(2):233-236

64. Meltvedt R Jr, Knecht B, Gibbons G, Stahler C, Stojowski $A$, Johansen $K$. Is nasogastric suction necessary after elective colon resection? Am J Surg 1985;149:620-622

65. Bashey AA, Cuschieri A. Patient comfort after upper abdominal surgery. J R Coll Surg Edinb 1985;30:97-100

66. Racette DL, Chang FC, Trekell ME, Farha GJ. Is nasogastric intubation necessary in colon operations? Am J Surg 1987;154:640-641
67. Cheatham ML, Chapman WC, Key SP, Sawyers JL. A meta-analysis of selective versus routine nasogastric decompression after elective laparotomy. Ann Surg 1995;221:469478

68. Nelson R, Tse B, Edwards S. Systematic review of prophylactic nasogastric decompression after abdominal operations. Br J Surg 2005;92:673-680

69. McAlister FA, Bertsch K, Man J, Bradley J, Jacka M Incidence of and risk factors for pulmonary complications after nonthoracic surgery. Am J Respir Crit Care Med 2005;171:514-517

70. Soop M, Carlson GL, Hopkinson J, et al. Randomized clinical trial of the effects of immediate enteral nutrition on metabolic responses to major colorectal surgery in an enhanced recovery protocol. Br J Surg 2004;91(9):11381145

71. Lewis SJ, Egger M, Sylvester PA, Thomas S. Early enteral feeding versus "nil by mouth" after gastrointestinal surgery: systematic review and meta-analysis of controlled trials. BMJ 2001;323(7316):773-776

72. Anderson HK, Lewis SJ, Thomas S. Early enteral nutrition within 24 hours of colorectal surgery versus later commencement of feeding for postoperative complications (review). Cochrane Database Syst Rev 2006;(4):CD004080

73. Wolff BG, Michelassi F, Gerkin TM, Techner L, Gabriel $\mathrm{K}, \mathrm{Du} \mathrm{W}$, Wallin BAand Alvimopan Postoperative Ileus Study Group. Alvimopan, a novel, peripherally acting $\mu$ opioid antagonist: results of a multicenter, randomized, double-blind, placebo-controlled, phase III trial of major abdominal surgery and postoperative ileus. Ann Surg 2004; 240(4):728-735

74. Delaney CP, Weese JL, Hyman NH, et al. Phase III trial of almivopan, a novel, peripherally acting mu opioid antagonist, for postoperative ileus after major abdominal surgery. Dis Colon Rectum 2005;48:1114-1129

75. Viscusi ER, Goldstein S, Witkowski T, et al. Alvimopan, a peripherally acting mu-opioid receptor antagonist, compared with placebo in postoperative ileus after major abdominal surgery; results of a randomized, double-blind, controlled study. Surg Endosc 2006;20:64-70

76. Jakobsen DH, Sonne E, Andreasson J, Kehlet H. Convalescence after colonic surgery with fast track versus conventional care. Scand J Surg 2004;93:24-28

77. Stephen AE, Berger DL. Shortened length of stay and hospital cost reduction with implementation of an accelerated clinical care pathway after elective colon resection. Surgery 2003;133:277-282

78. Basse L, Thorbol JE, Lossl K, Kehlet H. Colonic surgery with accelerated rehabilitation or conventional care. Dis Colon Rectum 2004;47(3):271-278

79. Gatt M, Anderson DG, Reddy BS, Hayward-Sampson P, Tring IC, MacFie J. Randomized clinical trial of multimodal optimization of surgical care in patients undergoing major colonic resection. Br J Surg 2005;92:1354-1362

80. Walter CJ, Smith A, Guillou P. Perceptions of the application of fast-track surgical principles by general surgeons. Ann R Coll Surg Engl 2006;88:191-195

81. Li S, Coloma M, White PF, et al. Comparison of the costs and recovery profiles of three anesthetic techniques for ambulatory anorectal surgery. Anesthesiology 2000;93: 1225-1230

82. Smith LE. Ambulatory surgery for anorectal diseases: an update. South Med J 1986;79(2):163-166 
83. Ferrera A, Gallagher J. The physician-owned ambulatory surgery center for colon and rectal surgery. In: Bailey HR, Snyder MJ, eds. Ambulatory Anorectal Surgery. New York: Springer; 1999:13-16

84. White PF. Update on ambulatory anesthesia. Can J Anaesth 2005;52(suppl 1):R1-R10

85. Gudaityte J, Marchertiene I, Pavalkis D. Anesthesia for ambulatory anorectal surgery. Medicina (Kaunas) 2004;40(2): 101-111

86. Liu SS, Strodtbeck WM, Richman JM, Wu CL. A comparison of regional versus general anesthesia for ambulatory anesthesia: a meta-analysis of randomized controlled trials. Anesth Analg 2005;101:1634-1642

87. Gepts E, Camu F, Cochshott ID, et al. Disposition of propofol administered as a constant rate intravenous infusion in humans. Anesth Analg 1987;66:1256-1263

88. Apfelbaum JL, Valawander CA, Gracela TH, et al. Eliminating intensive postoperative care in same day surgery patients using short-acting anesthetics. Anesthesiology 2002;97:66-74

89. Cousins $M$, Bridenbaugh $P$, eds. Neural Blockade in Clinical Anesthesia and Management of Pain. 2nd ed. Philadelphia: Lippincott; 1988

90. Rudkin GE. Local and regional anesthesia in the adult day surgery patient. In: Millar JM, Rudkin GE, Hitchcock M, eds. Practical Anesthesia and Analgesia for Day Surgery. Oxford: BIOS Scientific Publishers; 1997:207-210

91. Sa Rego MM, Watcha MF, White PF. The changing role of monitored anesthesia care in the ambulatory setting. Anesth Analg 1997;85:1020-1036

92. Marti MC. Anesthesie loco-regionale en chirurgie proctologique. Ann Chir 1993;47(3):250-255

93. Sun MY, Canete JJ, Friel JC, et al. Combination propofol/ ketamine is a safe and efficient anesthetic approach to anorectal surgery. Dis Colon Rectum 2006;49:1059-1065

94. Greco DP, Miotti G, Della Volpe A, Magistro C, De Carli S, Pugliese R. Stapled hemorrhoidopexy: day surgery or one day surgery? Surg Oncol 2007;16(suppl 1):S173-S175

95. Sayfan J, Becker A, Koltun L. Sutureless closed hemorrhoidectomy: a new technique. Ann Surg 2001;234(1):21-24

96. Longo A. Treatment of hemorrhoids disease by reduction of mucosa and hemorrhoidal prolapsed with a circular suturing device: a new procedure. Paper presented at: 6th World Congress of Endoscopic Surgery; June 3-6, 1998 Rome, Italy

97. Ceci F, Picchio M, Palimento D, Cali B, Corelli S, Spaziani E. Long-term outcome of stapled hemorrhoidopexy for grade III and grade IV hemorrhoids. Dis Colon Rectum 2008;51:1107-1112

98. Tjandra JJ, Chan MK. Systematic review on the procedure for prolapse and hemorrhoids (stapled hemorrhoidopexy). Dis Colon Rectum 2007;50(6):878-892

99. Jayaraman S, Colquhoun PH, Malthaner RA. Stapled versus conventional surgery for hemorrhoids. Cochrane Database Syst Rev 2006;(4):CD005393

100. Armstrong DN, Frankum C, Schertzer ME, Ambroze WL, Orangio GR. Harmonic scalpel hemorrhoidectomy. Dis Colon Rectum 2002;45(3):354-359

101. Kaidar-Person O, Person B, Wexner SD. Hemorrhoidal disease: a comprehensive review. J Am Coll Surg 2007;204(1): 102-117

102. Armstrong DN, Ambroze WL, Schertzer ME, Orangio GR. Harmonic scalpel vs. electrocautery hemorrhoidectomy: a prospective evaluation. Dis Colon Rectum 2001;44:558564

103. McCarus SD. Physiologic mechanism of the ultrasonically activated scalpel. J Am Assoc Gynecol Laparosc 1996;3: 601-608

104. Haveran LA, Sturrock PR, Sun MY, et al. Simple harmonic scalpel hemorrhoidectomy utilizing local anesthesia combined with intravenous sedation: a safe and rapid alternative to conventional hemorrhoidectomy. Int J Colorectal Dis 2007;22:801-806

105. Altomare DF, Milito G, Andreoli R, et al. Ligasure precise vs. conventional diathermy for Milligan-Morgan hemorrhoidectomy: a prospective, randomized, multicenter trial. Dis Colon Rectum 2008;51:514-519

106. Kwok SY, Chung CC, Tsui KK, Li MKW. A double-blind, randomized trial comparing Ligasure and Harmonic Scalpel hemorrhoidectomy. Dis Colon Rectum 2005;48:344-348

107. White PF. Droperidol: a cost effective antiemetic for over thirty years. Anesth Analg 2002;95:789-790

108. Souter AJ, Fredman B, White PF. Controversies in the perioperative use of nonsteroidal anti-inflammatory drugs. Anesth Analg 1994;79:1178-1190

109. Ding Y, White PF. Comparative effects of ketorolac, dezocine and fentanyl as adjuvants during outpatient anesthesia. Anesth Analg 1992;75:566-571

110. Coloma M, White PF, Huber PJ, Tongier WK, Dullye KK, Duffy LL. The effect of ketorolac on recovery after anorectal surgery: intravenous versus local administration. Anesth Analg 2000;90:1107-1110

111. O'Donovan S, Ferrara A, Larach S, Williamson P. Intraoperative use of Toradol facilitates outpatient hemorrhoidectomy. Dis Colon Rectum 1994;37(8):793-799

112. Place RJ, Coloma M, White PF, Huber PJ, Van Vlymen J, Simmang CL. Ketorolac improves recovery after outpatient anorectal surgery. Dis Colon Rectum 2000;43(6):804-808

113. Tang J, Wang B, White PF, et al. Effect of timing of ondansetron administration on its efficacy, cost-effectiveness, and cost benefit as a prophylactic antiemetic in the ambulatory setting. Anesth Analg 1998;86:274-282

114. Coloma M, Duffy LL, White PF, Tongier WK, Huber PJ Jr. Dexamethasone facilitates discharge after outpatient anorectal surgery. Anesth Analg 2001;92:85-88

115. Henzi I, Walder B, Tramer MR. Dexamethasone for the prevention of postoperative nausea and vomiting: a quantitative systematic review. Anesth Analg 2000;90:186-194

116. Aasboe V, Raeder JC, Groegaard B. Betamethasone reduces postoperative pain and nausea after ambulatory surgery. Anesth Analg 1998;87:319-323

117. Scuderi PE, James RL, Harris L, Mimms GR. Multimodal antiemetic management prevents early postoperative vomiting after outpatient laparoscopy. Anesth Analg 2000;91: 1408-1414

118. Yogendran S, Asokumar B, Cheng D, Chung F. A prospective, randomized double-blind study of the effect of intravenous fluid therapy on adverse outcomes after outpatient surgery. Anesth Analg 1995;80:682-686

119. Prasad ML, Abcarian H. Urinary retention following operations for benign anorectal diseases. Dis Colon Rectum 1978;21(7):490-492

120. Toyonaga T, Matsushima M, Sogawa N, et al. Postoperative urinary retention after surgery for benign anorectal disease: potential risk factors and strategy for prevention. Int J Colorectal Dis 2006;21(7):676-682 
121. Petros JG, Bradley TM. Factors influencing postoperative urinary retention in patients undergoing surgery for benign anorectal disease. Am J Surg 1990;159:374-376
122. Bailey HR, Ferguson JA. Prevention of urinary retention by fluid restriction following anorectal operations. Dis Colon Rectum 1976;19:250-252 